Flora-Fauna-Habitat-Klage der EU

\section{Hausaufgaben nicht gemacht}

Naturnahes Grünland ist für die Erhaltung der Biodiversität sowie für zahlreiche Ökosystemleistungen essenziell. Grünlandstandorte bieten Lebensräume für eine Vielzahl von Tier- und Pflanzenarten. Aufgrund der ganzjährigen Bedeckung verfügt Grünland über hohe Humusgehalte und eine hohe Wasserspeicherkapazität. Es bietet Schutz gegenüber Wind- und Wassererosion. Im Randbereich von Gewässern kann Grünland den Eintrag von Nähr- und Schadstoffen aus angrenzenden Flächen in die Gewässer vermindern. Der hohe Kohlenstoffgehalt im Boden hat positive Wirkungen für den Klimaschutz. Der Anteil des Grünlands an der landwirtschaftlich genutzten Fläche nahm in Deutschland in der Vergangenheit stetig ab. Während 1991 noch über 5,3 Mio. ha als Dauergrünland bewirtschaftet wurden, betrug diese rund 15 Jahre später nur noch ca. 4,6 Mio. ha. Artenreiches Grünland mit hohem naturschutzfachlichen Wert (High-Nature-ValueGrünland) ist davon besonders betroffen: Zwischen 2009 und 2015 gingen diese Flächen bundesweit um 8,9\% zurück. Seitdem ist die Rate des Rückgangs gering, aber an vielen Standorten verschlechtert sich das vorhandene Grünland weiter. Auf Wiesen und Weiden sind laut Naturschutzbund Deutschland (NABU) daher mittlerweile $80 \%$ der Lebensräume für Pflanzen und Tiere in Gefahr, 35\% sind von der vollständigen Vernichtung bedroht.

Aus gutem Grunde wurde Grünland daher unter Schutz gestellt. Die 1992 verabschiedete Flora-Fauna-Habitat (FFH-)Richtlinie der EU ist hier ein wichtiges Element. Mit der Richtlinie sollen Pflanzen und Tiere im Rahmen eines gebietsbezogenen Naturschutzes erhalten werden. Damit wurde Deutschland unter anderem verpflichtet, sowohl im Bergland als auch im Flachland blütenreiche Wiesen als Natura-2000-Schutzgebiete auszuweisen und durch angepasste Bewirtschaftung zu schützen.

Die EU hat nun Deutschland wegen Vertragsverletzungsverfahren beim Europäischen Gerichtshof angeklagt. Was steckt dahinter? Die EU argumentiert, dass die Bundesrepublik beim FFH-Naturschutz ihren Verpflichtungen nicht nachgekommen sei. Dies bezieht sich im Wesentlichen auf vier Aspekte: Erstens sei die rechtlich verbindliche Sicherung von FFH-Schutzgebieten unzureichend. Zweitens seien ausreichend qualifizierte, gebietsspezifische Erhaltungsziele (immer noch) nicht definiert worden. Die-

(C) Der/die Autor:in(nen) 2021. Open Access: Dieser Artikel wird unter der Creative Commons Namensnennung 4.0 International Lizenz veröffentlicht (creativecommons.org/licenses/by/4.0/deed.de).

Open Access wird durch die ZBW - Leibniz-Informationszentrum Wirtschaft gefördert. se Ziele sollten auch quantitativ festgelegt sowie messund überprüfbar sein. Drittens seien die gebietsspezifischen Maßnahmenprogramme zur Erhaltung des Grünlands zu schwach ausgestaltet, um dem drängenden Ziel des Stopps des Biodiversitätsverlustes zu begegnen. Und viertens schließlich würde den Öffentlichkeits- und Informationspflichten in einigen Bundesländern nicht ausreichend Rechnung getragen. Deutschland verweist hingegen darauf, dass bereits viel für die Umsetzung der Richtlinie getan worden sei, sowohl auf Bundesebene beim Schutz der Außerordentlichen Wirtschaftszone (AWZ) in der Nord- und Ostsee als auch auf Ebene der Bundesländer mit ihren bisherigen Fortschritten in der Zielerreichung. Hinsichtlich der Ausweisung der Schutzgebiete sei zudem eine prozentual hohe Rate erreicht worden.

Die Klage der EU-Kommission kommt keineswegs überraschend. Der Streit brodelt schon lange und zieht sich hin. Nachdem die Verpflichtungen zur Ausweisung und Sicherung der FFH-Gebiete bereits ab 2010 fällig waren, und es schon zu der Zeit Diskussionen zwischen der EU-Kommission und Deutschland gab, wurde das Beschwerdeverfahren offiziell 2015 eingeleitet. 2019 erfolge ein ergänzendes Mahnschreiben der EU und im Herbst 2020 dann eine „Begründete Stellungnahme“, in der die Kommission erneut ausführlich ihre rechtliche und fachliche Position darlegte. So gesehen ist die jetzige Klage ein nahezu absehbarer nächster Schritt gewesen. Deutschland hat seine Hausaufgaben im Naturschutz nicht gemacht und zugesagte Fristen nicht eingehalten, zum Teil auch weil die EUVorgaben auf deutscher Seite anders interpretiert werden. Die Kommission ist nach den vielen „Warnschüssen“ nicht mehr gewillt gewesen, sich weiter vertrösten zu lassen.

Die Entwicklung zeigt wieder einmal, dass Deutschland nicht zu den Musterschülern im Umwelt- und Naturschutz in Europa zählt. Immer wieder steht dabei die Landwirtschaft im Vordergrund. Bei der FFH-Klage sind es unter anderem nicht nachhaltige landwirtschaftliche Bewirtschaftungsweisen in den Natura-2000-Gebieten. Nach der EU-Klage wegen der Nicht-Umsetzung der EU-Wasserrichtlinie aufgrund zu hoher Nitrateinträge ist dies nun ein weiterer Fall der Auseinandersetzungen. Dies dürfte Deutschland nicht nur allein wegen der drohenden empfindlichen Strafzahlungen hart treffen. Wichtiger erscheint, dass hierzulande immer noch unzureichend anerkannt ist, dass der Verlust wertvollen Naturkapitals auch ökonomisch eine Rolle spielt. Denn Grünlandflächen zu verlieren, ist letztlich ein gesellschaftliches Verlustgeschäft, wie das Vorhaben Naturkapital Deutschland gezeigt hat.

Bernd Hansjürgens Helmholtz-Zentrum für Umweltforschung - UFZ bernd.hansjuergens@ufz.de 